\title{
Researching turbulent convection models and the density gradient reversing
}

\author{
Q. S. Zhang ${ }^{1,2}$ and Y. Li ${ }^{1}$ \\ ${ }^{1}$ National Astronomical Observatories/Yunnan Observatory, Chinese Academy of Sciences, PO \\ Box 110, Kunming 650011, China \\ ${ }^{2}$ Graduation School of Chinese Academy of Sciences, Beijing 100039, China \\ Email: mail_aoe_163@.com
}

\begin{abstract}
Turbulent convection models (TCM) provide a better way to study convection in stars than the MLT. Improving numerical method, we adopted larger diffusion parameters and smaller dissipation parameters in order to further correct the p-mode oscillation frequencies of TCM models. The density gradient reversing is discussed.
\end{abstract}

Keywords. convection, hydrodynamics, stars: interiors

\section{TCM Solar models}

Li and Yang (2007) tested the TCM, and found the p-mode oscillation frequencies of their TCM solar models are in better agreement with the observation than those of MLT models. It is expected that the p-mode oscillation frequencies of such models can be further improved by increasing diffusion parameters and decreasing dissipation parameters of the TCM they have adopted(Yang \& Li, 2007). However, numerical problems precluded them from getting better model. After improving numerical method, we got better models. Table 1 shows solar models we calculated, they will be discussed in this paper.

\section{The p-mode oscillation frequencies}

Figures 1 and 2 show the frequency differences of p-mode oscillations with the spherical harmonic index $l=3,150$. It is obvious that differences of p-mode oscillations frequency of NLSM solar models are reduced more than 30\% compared with those of SSM almost in all frequency range and for different $l$. Our results are better than those obtained by Yang and Li (2007) and so confirm the judgment that the p-mode oscillation frequencies of solar models with the TCM can be further improved by increasing diffusion parameters and decreasing dissipation parameters. Those results show the advantage of TCM.

Another important result is the appearance of density gradient reversing in our models, which is not found in the SSM. This result makes the relation of TCM's parameter and the density gradient reversing to be a more important and more interesting topic.

Table 1. The information of model parameters

\begin{tabular}{lcccccccc}
\hline Model & $C_{t}$ & $C_{e}$ & $C_{k}$ & $C_{t 1}$ & $C_{e 1}$ & $C_{s}$ & $\alpha$ & $Y_{0}$ \\
\hline NLSM $(1)$ & 0.25 & 0.10 & 2.50 & 0.11 & 0.20 & 0.05 & 0.05852 & 0.27509 \\
NLSM $(2)$ & 0.20 & 0.10 & 2.50 & 0.15 & 0.25 & 0.10 & 0.05162 & 0.27509 \\
NLSM $(3)$ & 3.00 & 1.25 & 2.50 & 0.0313 & 0.0313 & 0.0313 & 0.8966 & 0.27506 \\
NLSM $(4)$ & 2.00 & 1.00 & 2.50 & 0.0277 & 0.0277 & 0.0277 & 0.6305 & 0.27506 \\
NLS $M(5)$ & 1.70 & 0.85 & 2.50 & 0.0205 & 0.0205 & 0.0205 & 0.5339 & 0.27506 \\
S S M & - & - & - & - & - & - & 1.642 & 0.27506 \\
\hline
\end{tabular}




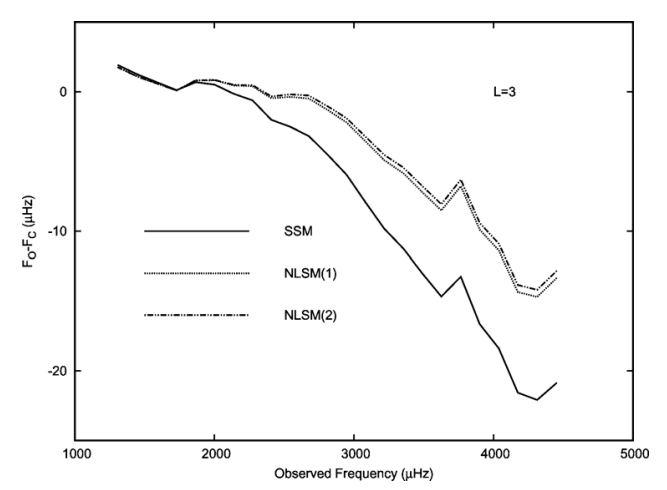

Figure 1. O-C of p-mode oscillation frequencies with $l=3$.

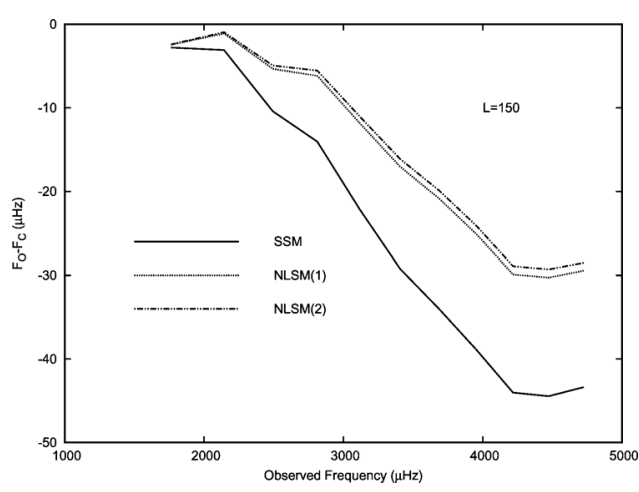

Figure 2. O-C of p-mode oscillation frequencies with $l=150$.

\section{Density gradient reversing}

The density gradient reversing is impossible in radiative equilibrium zone for the maximum of gravitational potential. We found that density gradient reversed in the convection zone if $\nabla>\nabla_{\rho}=\left(\frac{\partial \ln T}{\partial \ln P}\right)_{\rho}$ which is the criterion of it. Because $\nabla_{\rho}>\nabla_{a d}$, the reversing does in superadiabatic convection zone only if it does.

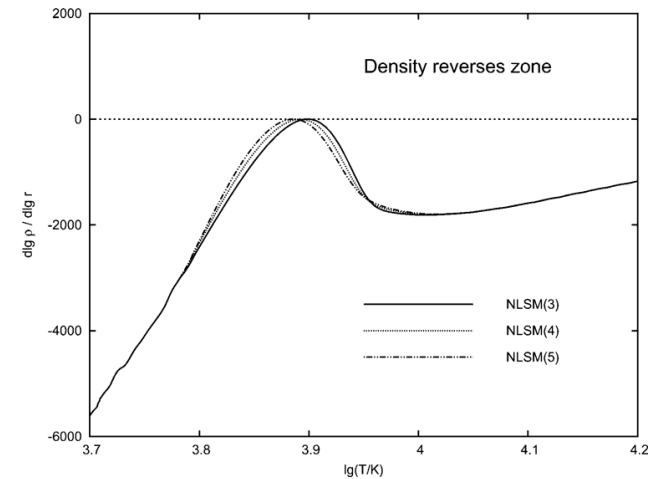

Figure 3. The density gradient of critical models in convection zone.

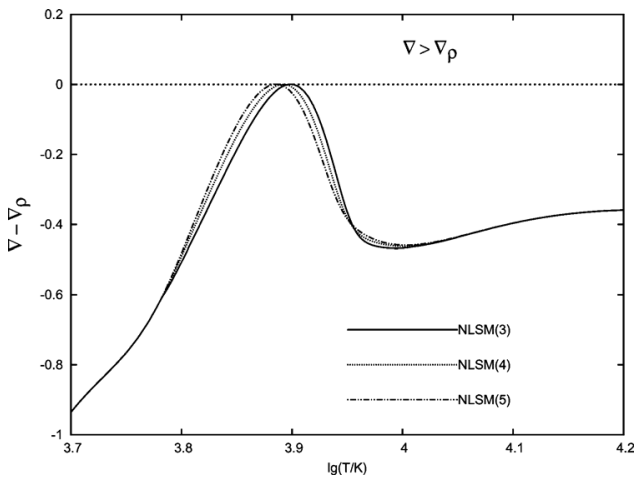

Figure 4. $\nabla-\nabla_{\rho}$ of critical models in convection zone.

We got some critical solar models(NLSM(3), $\operatorname{NLSM}(4)$ and $\operatorname{NLSM}(5)$, see table 1) of the density gradient reversing, Figure 4 shows their reversing density gradient, Figure 5 shows $\nabla_{\rho}>\nabla_{a d}$ of each model and validates the criterion. It can be found from table 1 that the density gradient reversing is dominated by the diffusion parameters $C_{t 1}, C_{e 1}$ and $C_{s}$, because the $\nabla$ increase if we enlarged those parameters(Li \& Yang, 2007).

\section{References}

Li, Y. \& Yang, J. Y., 2007, MNRAS, 375, 388

Yang, J. Y. \& Li, Y., 2007, MNRAS, 375, 403 